\title{
Effects of Transcutaneous Electrical Acupoint Stimulation (TEAS) on Postoperative Recovery in Patients with Gastric Cancer: A Randomized Controlled Trial
}

This article was published in the following Dove Press journal:

Cancer Management and Research

\author{
Xin Zhou' \\ Shou-Gen Cao' \\ Xiao-Jie Tan' \\ Xiao-Dong Liu' \\ Ze-Qun $\mathrm{Li}^{1}$ \\ Ling-Xin Kong ${ }^{2}$ \\ Yu-Long Tian' \\ Dan Liu' \\ Shuai Shen' \\ Yu-Qi Sun' \\ Hai-Tao Jiang ${ }^{\prime}$ \\ Yan-Bing Zhou' \\ 'Department of Gastrointestinal Surgery, \\ The Affiliated Hospital of Qingdao \\ University, Qingdao, Shandong Province, \\ People's Republic of China; ${ }^{2}$ Department \\ of Rehabilitation, The Affiliated Hospital \\ of Qingdao University, Qingdao, \\ Shandong Province, People's Republic of \\ China
}

Purpose: Transcutaneous electrical acupoint stimulation (TEAS) is an innovative choice for postoperative pain management. However, the safety and effectiveness of this traditional Chinese medicine (TCM) therapy for patients who underwent gastrectomy is largely unknown. So, the purpose of this study is to evaluate the safety and effectiveness of TEAS for patients who underwent gastrectomy.

Patients and Methods: We recruited 96 patients with gastric cancer from May 2019 to November 2019; 82 patients were enrolled, and 81 patients completed. Patients were randomly assigned to TEAS group (TG) received TEAS on postoperative day (POD) 1-3 or control group (CG) at a 1:1 ratio. The primary outcomes were pain score and consumption of analgesics. The secondary were the time of first postoperative flatus and defecation, frequency of postoperative nausea, vomiting, distention, diarrhea, comfort of semi-fluid diet, Clavien-Dindo grade (C-D grade) and length of postoperative day. We performed hematological analysis to explore the possible mechanisms.

Results: Overall, 81 patients were enrolled included in the analysis. Compared with $\mathrm{CG}$, pain scores in TG were lower on POD $1-5$ (average: $2.55 \pm 0.21$ vs $3.10 \pm 0.42, P<0.001$ ), and the use rate of opioids was lower (43.9 vs 75.0, $P=0.004$ ); time of first postoperative flatus $(55.63 \pm 16.74$ vs $72.60 \pm 20.92, P<0.001)$ and defecation $(72.20 \pm 16.24$ vs $95.78 \pm 17.75$, $P<0.001)$ were shorter; the frequency of nausea were fewer $(1.88 \pm 1.09$ vs $2.58 \pm 0.77$, $P=0.029)$ and patients were more comfortable with semi-fluid diet $(7.63 \pm 0.63$ vs 6.93 $\pm 0.69, P<0.001$ ); among the hematologic results, $\beta$-endorphin ( $\beta$-End), interleukin-2 (IL-2), motilin (MTL) on POD 3, POD 5 were lower, 5-hydroxytryptamine (5-HT), interleukin-6 (IL-6), tumor necrosis factor- $\alpha$ (TNF- $\alpha$ ) were higher. And no adverse event was reported.

Conclusion: TEAS can relieve postoperative pain and promote the recovery of gastrointestinal function. Consequently, it can be an adjunctive therapy to enhance postoperative recovery for patients after gastrectomy.

Keywords: stomach neoplasms, acupuncture therapy, enhanced recovery after surgery, pain management, gastrointestinal function

\section{Introduction}

In 2018, gastric cancer was the fifth most prevalent cancer and ranked third in cancer-related mortality. ${ }^{1}$ Surgery is the only curative treatment for advanced gastric cancer and D2 lymphadenectomy is recognized as the standard procedure
Correspondence: Yan-Bing Zhou Department of Gastrointestinal Surgery, The Affiliated Hospital of Qingdao University, No. 16 Jiangsu Road, Shinan District, Qingdao City, Shandong Province, People's Republic of China Tel +86-13708971773

Email zhouyanbing999@aliyun.com
Cancer Management and Research 2021:13 |449-1458

submit your manuscript DovePress $f y$ in $\boldsymbol{v}$ http://doi.org/10.2147/CMAR.S292325 
Asia-wide. ${ }^{2}$ Regardless of the approach of surgery, there must be pain following surgical injury, ${ }^{3}$ which may be mediated by afferent nervous stimulation together with complex inflammatory-immunological responses. ${ }^{4,5}$ Enhanced recovery after surgery (ERAS), a perioperative concept proposed by professor Kehlet in $1997,{ }^{6}$ has been introduced worldwide ever since and its ultimate purpose is to modify undesirable stress ${ }^{7}$ and pain management is one essential evidence-based component in this multimodal care program ${ }^{8}$ to establish a "fast track" recovery pathway. Whether it is inflammatory pain or neuropathic pain it will cause kinds of complex problems not only in cardiopulmonary system. ${ }^{9}$ That's why we prioritize postoperative pain commonly aiming at lessening suffering and shortening convalescence. Opioid is a cornerstone in clinical analgesia though adverse outcomes such as respiratory depression, misuse have attracted out attentions in recent years. In pain management, multimodal analgesia emphasizes the combination of analgesics with different mechanisms and techniques to minimize the opioids use and side effects. ${ }^{10}$ In fact, in addition to new drugs with lower side effects, novel techniques are being explored in many medical centers.

Acupuncture is an ancient empirical medicine in traditional Chinese medicine (TCM). Nowadays, it has been demonstrated that acupuncture can be of benefit to pain control. ${ }^{11}$ Ancient physicians believed that Yin and Yang are interactive and interdependent opposing forces universally, referring cold and warm, static and active, Qi and blood (in body) ${ }^{12}$ (Appendix 1). And acupuncture corrects the imbalance of Yin and Yang by readjusting the body's meridians which are energy-carrying pathways supporting the circulation of Qi and blood. Nowadays, acupuncture has derived new forms, such as electroacupuncture (EA), transcutaneous electrical acupoint stimulation (TEAS), etc. Among them, TEAS evolved on the basis of EA with similar efficacy ${ }^{13}$ and some studies claimed that it can enhance rehabilitation. ${ }^{14,15}$ Due to the non-invasive and convenience of TEAS, it has been experimented to improve recovery in various subjects. ${ }^{16,17}$ However, there is still a need for more high-quality researches on the perioperative application of TEAS. So, the objective of this trial was to validate the efficacy of TEAS on postoperative recovery in patients after gastrectomy. In addition, we also hope to explore molecular interpretations of TEAS in this trial.

\section{Patients and Methods}

\section{Trial Design}

This was an unblinded randomized controlled trial. The protocol was approved by the ethics committee of the Affiliated Hospital of Qingdao University, and its ethical approval number is QYFYKYLL201812. It was carried out at the Department of Gastrointestinal Surgery, the Affiliated Hospital of Qingdao University, between May 1, 2019 and October 31, 2019. A total of 96 patients were recruited. Eighty-two patients who were eligible for inclusion criteria were randomly assigned into TEAS group (TG) receiving 3-consecutive-day TEAS and control group (CG) at a 1:1 ratio. During the perioperative period, the same ERAS protocol (See Appendix 2 for detail) was performed on both groups of patients who underwent standard laparoscopic/robot radical gastrectomy. We collected clinical data and blood samples for analysis. This trial has been registered and reviewed at the Chinese Clinical Trial Registry with registration number ChiCTR1900022692. The date of trial registration was April 22, 2019. This study was conducted in accordance with the Declaration of Helsinki.

Please contact 215099754@qq.com to view the full trial protocol.

\section{Participants}

The inclusion, exclusion and withdraw criteria were as follows. Inclusion: 1. Any religion or gender; 2. 18-75 years old; 3. Proposed radical surgery for gastric cancer (laparoscopy/robot); 4. Signed the informed consent. Exclusion: 1. Received neoadjuvant therapy; 2. American Society of Anesthesiologists (ASA) grade $>3$; 3. cTNM stage IV (AJCC-American Joint Committee on Cancer, 8th Edition Cancer Staging); 4. Current or previous diabetes; 5. Systemic infection or contagious disease; 6 . Chronic constipation or diarrhea; 7. Current or previous cardiovascular/cerebrovascular accident or central nervous system injury; 8 . Mental incapacity or severe emotional or mental illness; 9. Cardiac pacemaker; 10. Local infiltration anesthesia for incision; 11. Anesthesia is not epidural anesthesia-assisted general anesthesia; 12 . Use of patientcontrolled analgesia (PCA) after surgery. Withdraw: 1. Severe accident occurred during the operation such as hemorrhagic shock, cardiogenic shock, ventricular fibrillation; 2. Severe comorbidities after surgery such as pulmonary embolism, severe pneumonia; 3 . Patients or family members decide to withdraw. It should be noted that the 
Clavien-Dindo grade (C-D grade) was used for postoperative comorbidity grade and patients with a grade $>2$ met the withdrawal criteria.

\section{Intervention}

We selected several acupoints with potential functions of pain relief and promoting gastrointestinal recovery. According to the WHO standard acupuncture point locations (Appendix 3), the chosen acupoints are as follows: 1. Hegu (LI4): On the dorsum of the hand, between the first and second metacarpal bone, radial to the midpoint of the second metacarpal bone. 2. Neiguan (PC6): On the anterior aspect of the forearm, between the tendons of the palmaris longus and the flexor carpi radialis, 2 cun proximal to the palmar wrist crease. 3. Weishu (BL21): In the upper back region, at the same level as the inferior border of the spinous process of the 12th thoracic vertebra (T12), 1.5 cun lateral to the posterior median line. 4 . Xiaochangshu (BL27): In the sacral region, at the same level as the first posterior sacral foramen, and 1.5 cun lateral to the median sacral crest. 5. Zusanli (ST36): On the anterior aspect of the leg, on the line connecting ST35 (on the anterior aspect of the knee, in the depression lateral to the patellar ligament) with ST41 (on the anterior aspect of the ankle, in the depression at the center of the front surface of the ankle joint, between the tendons of extensor hallucis longus and extensor digitorum longus), 3 cun inferior to ST35. 6. Shangjuxu (ST37): On the anterior aspect of the leg, on the line connecting ST35 with ST41, 6 cun inferior to ST35.

For patients in TG, they received TEAS therapy at 8:00 a.m. and 4:00 p.m. on postoperative day (POD) 1-3 lasting 30 minutes each time. After sterilizing the skin surrounding the acupoints with $75 \%$ alcohol, we applied the electrode pads on the acupoints.

\section{Devices}

The device we used was Huatuo Electronic Acupuncture Treatment Instrument SDZ-II (Suzhou Medical Supplies Factory Co., Ltd., Registration number 20172270675), and the frequency was set on $20 / 100 \mathrm{~Hz}(20 \mathrm{~Hz} 5 \mathrm{~s}, 100 \mathrm{~Hz}$ $10 \mathrm{~s})$. The other parameters such as intensity are set according to the actual experience of the patients, taking the patient's discomfort as the upper limit.

\section{Outcomes}

The primary outcomes of this trial were as follows: (1) Pain score. We used numerical rating scale (NRS, in 10 levels and we have improved the basic unit by dividing the 10 levels into 20 levels, ie, 0.5 as a unit) combined with the Wong-Baker faces pain rating scale. We recorded the pain score at 6-time points (last night till 8:00, 8:00, 12:00, 16:00, 20:00, time before sleep) a day from POD 1 to POD 5. On POD 0, we recorded the pain scores at the awake time postoperatively and other time points at the above. (2) Consumption of analgesics. Non-steroidal antiinflammatory drugs (NSAIDs) were used for mild to moderate pain, and opioids were used for moderate to severe pain. We used NSAIDs (flurbiprofen axetil) once on POD 1 regularly and then we made prescriptions according to the conditions of patients. And we recorded consumption of NSAIDs by doses and opioids by equivalent doses of morphine (pethidine: pentazocine: dezocine: morphine=100mg: $30-60 \mathrm{mg}: 10 \mathrm{mg}: 10 \mathrm{mg}$ ). It should be noted that all patients enrolled did not use PCA.

The secondary outcomes were as follows: time of first postoperative flatus and defecation, frequency of postoperative nausea, vomiting, distention, diarrhea, fever, comfort of semi-fluid diet (patient subjective score, range 0-10), Clavien-Dindo grade and length of postoperative day. For definition of fever, we set $37.3^{\circ} \mathrm{C}$. And we measured the temperature 6 times a day and recorded it once if it appears.

We collected patients' peripheral venous blood on POD 1, POD 3 and POD 5 for analysis. White blood cell count (WBC), percentage of neutrophils (NEC\%), C-reactive protein (CRP) and procalcitonin (PCT) were measured with plasma; $\beta$ endorphin ( $\beta$-End), 5-hydroxytryptamine (5-HT), interleukin2 (IL-2), interleukin-6(IL-6), tumor necrosis factor- $\alpha$ (TNF- $\alpha$ ), motilin (MTL) were measured with serum (serum samples were centrifuged at $2000 \mathrm{~g}$ for $10 \mathrm{~min}$, and the serum was stored at $-80^{\circ} \mathrm{C}$ until analysis) by using enzyme-linked immunosorbent assay (ELISA). The kit was purchased from Jiangsu Yutong Biological Technology Co., Ltd.

All frequency-type outcomes (such as nausea, vomiting frequency, etc.) were collected by querying patients at 8:00, 12:00, 18:00 daily and it was recorded once there was an appearance. All data were recorded by Yulong Tian and Zequn Li.

\section{Sample Size}

The sample size justification of this trial was based on the preliminary data of pain scores which was from a pilot study we previously performed in our center. The results of the pilot study showed that the average pain score of POD 1-5 were $2.80 \pm 0.45$ and $3.20 \pm 0.72$ for TG and CG groups, respectively. With an equal randomization and assuming the dropout rate 
was less than $10 \%$, a sample size of 82 patients (41 in the TG and 41 in the CG) will ensure the power was at least $80 \%$ to detect the difference at a significant level of 0.05 , using a twosided $t$-test. We estimated it would need at least 6 months to enroll all cases.

\section{Randomization}

We randomized patients according to the order of the random number table generated by SPSS 21.0 (SPSS, Inc., Chicago, IL, USA). We determined the random allocation sequence according to the time when patients signed the informed consent for anesthesia, and Yuqi Sun was responsible for allocating the patients according to the random number table.

\section{Statistical Methods}

All statistical analysis was performed using SPSS 21.0 and Graphpad Prism 8.0.2 (GraphPad Software, Inc., La Jolla, CA, USA). The level of significance for all statistical tests was set at $0.05(\alpha=0.05)$. Data are presented as means and standard deviations (Mean \pm SD) for continuous variables and as proportions for categorical variables. Median and quartile were used in skewed distribution data. The continuous variables were assessed using Student's $t$-test when Gaussian distribution is verified. Otherwise Wilcoxon rank sum test will be used alternatively. The association between categorical variables were assessed using Chi-squared test $\left(X^{2}\right)$. The ordinal variables were assessed using Wilcoxon rank sum test. Analysis of variance will be used if Gaussian distribution is verified in repeated measurement data; if not, the generalized estimating equation will be used. A two-tailed $P$ value of $<0.05$ was regarded as statistically significant.

\section{Results}

\section{Baseline Data}

The participant flow diagram is shown in Figure 1. The clinicopathological characteristics and surgical outcomes

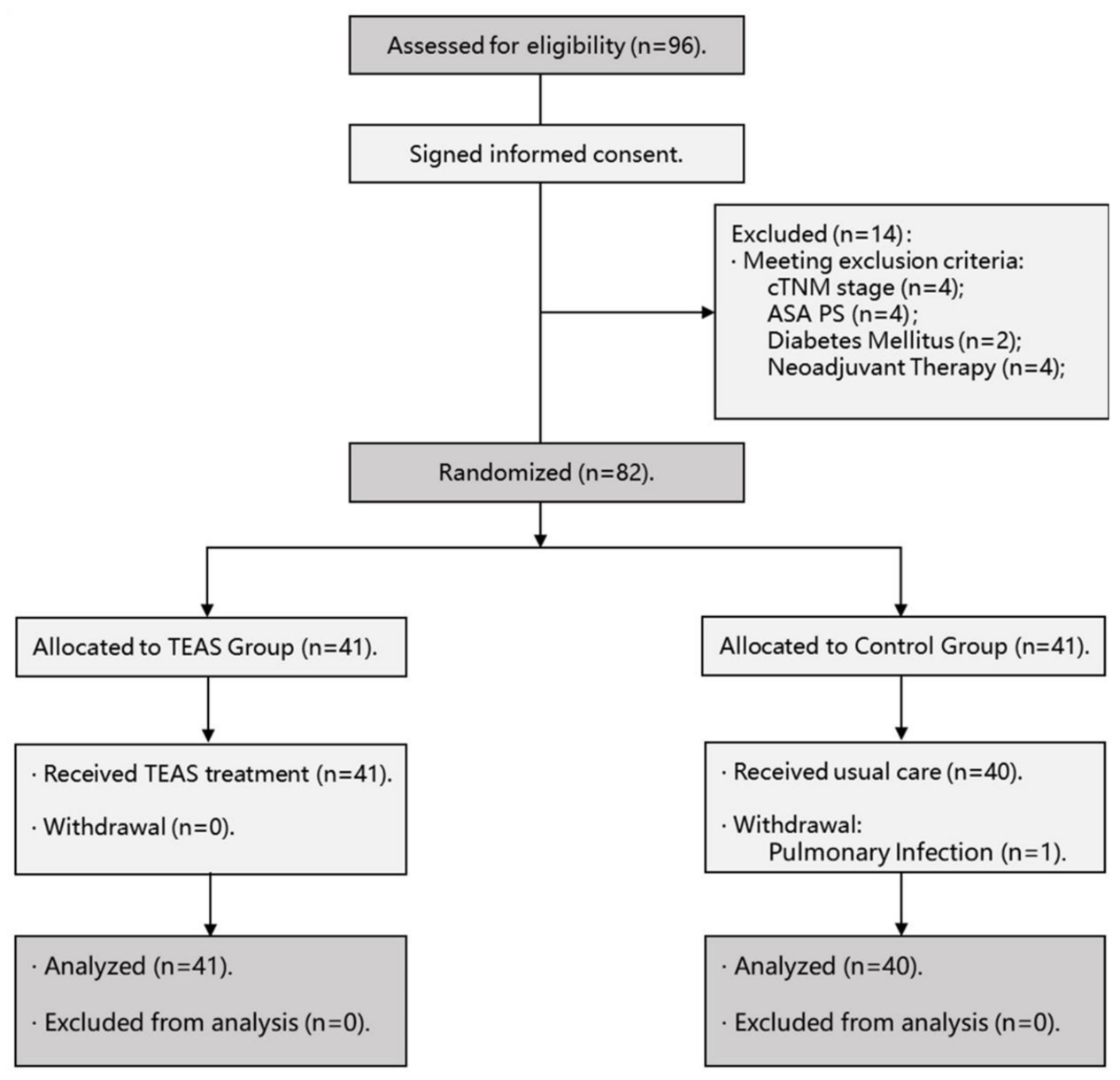

Figure I Participant flow diagram.

Abbreviation: ASA, American Society of Anesthesiologists. 
of patients were shown in Tables 1 and 2, and there were no significant differences between 2 groups $(P>0.05)$. Additionally, the anesthesiologists of all patients came

Table I Clinicopathological Characteristics of Patients $(n=8 I)$

\begin{tabular}{|c|c|c|}
\hline & TG & CG \\
\hline & $(n=4 I)$ & $(n=40) P$ \\
\hline Age (years) & & $0.304^{\mathrm{a}}$ \\
\hline Mean $\pm S D$ & $58.7 I \pm 9.274$ & $60.83 \pm 9.137$ \\
\hline Gender & & $0.689^{b}$ \\
\hline Male, n (\%) & $27(65.9)$ & $28(70.0)$ \\
\hline Female, n (\%) & $14(34.1)$ & $12(30.0)$ \\
\hline BMI $\left(\mathrm{kg} / \mathrm{m}^{2}\right)$ & & $0.988^{\mathrm{a}}$ \\
\hline Mean \pm SD & $23.93 \pm 2.823$ & $23.93 \pm 2.696$ \\
\hline ASA grade, n (\%) & & $0.310^{c}$ \\
\hline 1 & $0(0.00)$ & $0(0.00)$ \\
\hline 2 & $35(85.4)$ & $37(92.5)$ \\
\hline 3 & $6(14.6)$ & $3(7.50)$ \\
\hline $\mathrm{CCl}$ & & $0.342^{c}$ \\
\hline Mean \pm SD & $3.95 \pm 1.26$ & $4.20 \pm 1.20$ \\
\hline NRS 2002 score & & $0.359^{c}$ \\
\hline Mean $\pm S D$ & $1.65 \pm 1.85$ & $2.05 \pm 1.80$ \\
\hline \multirow[t]{2}{*}{ Smoker, n (\%) } & & $0.585^{b}$ \\
\hline & $24(58.5)$ & $21(52.5)$ \\
\hline \multirow[t]{2}{*}{ Drinker, n (\%) } & & $0.586^{b}$ \\
\hline & $15(36.6)$ & $17(42.5)$ \\
\hline \multirow[t]{2}{*}{ Abdominal pain, n (\%) } & & $0.882^{b}$ \\
\hline & $26(63.4)$ & $26(65.0)$ \\
\hline \multirow[t]{2}{*}{ Abdominal distention, $\mathrm{n}(\%)$} & & $0.094^{b}$ \\
\hline & $13(31.7)$ & $20(50.0)$ \\
\hline \multirow[t]{2}{*}{ Diarrhea, n (\%) } & & $0.980^{b}$ \\
\hline & $2(4.90)$ & $2(5.00)$ \\
\hline \multirow[t]{2}{*}{ Constipation, n (\%) } & & $0.667^{b}$ \\
\hline & $3(7.30)$ & $4(10.0)$ \\
\hline \multirow[t]{2}{*}{ Nausea, n (\%) } & & $0.503^{b}$ \\
\hline & $13(31.7)$ & $10(25.0)$ \\
\hline \multirow[t]{2}{*}{ Vomiting, n (\%) } & & $0.753^{b}$ \\
\hline & $5(12.2)$ & $4(10.0)$ \\
\hline \multirow[t]{2}{*}{ Appetite loss, n (\%) } & & $0.558^{b}$ \\
\hline & $18(43.9)$ & $15(37.5)$ \\
\hline cTNM stage, n (\%) & & $0.807^{c}$ \\
\hline 1 & $18(43.9)$ & $16(40.0)$ \\
\hline II & $7(17.1)$ & $8(20.0)$ \\
\hline III & $16(39.0)$ & $16(40.0)$ \\
\hline
\end{tabular}

Notes: ${ }^{\mathrm{a}}$ t-test; ${ }^{\mathrm{b}} \mathrm{Chi}$-squared test $\left(X^{2}\right)$; ${ }^{\mathrm{C}}$ Wilcoxon rank sum test.

Abbreviations: BMI, body mass index; ASA, American Society of Anesthesiologists; $\mathrm{CCl}$, Charlson Comorbidity Index; NRS, nutrition risk screening. from the same anesthesia team, and the use of anesthetic drugs during the operation should be as consistent as possible. See Appendix 4 for details.

\section{Primary Outcomes}

Among the primary outcomes (Table 3 ), there was no difference in pain scores of 2 groups on POD $0(P=0.105)$. The pain scores of TG were significantly lower than CG on POD $1-5(P<0.001)$. As shown in Figure 2, the use rate of opioids was significantly lower in TG $(P=0.004)$.

\section{Secondary Outcomes}

Among the secondary outcomes (Table 4), the time of first postoperative flatus was shorter in TG $(P<0.001)$, so as the time of first postoperative defecation $(P<0.001)$. And the comfort of semi-fluid diet of TG was higher than that of CG $(P<0.001)$. For the frequency of symptoms, postoperative nausea was significantly less in TG $(P=0.029)$.

\section{Hematological Results}

As can be seen from Table 5, on POD 3 and POD 5, $\beta$ End, IL-2, MTL were significantly higher and 5-HT, IL-6, TNF- $\alpha$ were significantly lower in $\mathrm{TG}$ than those in $\mathrm{CG}$ $(P<0.001)$.

\section{Data Sharing Statement}

The original data of this study will not be shared publicly. If necessary, please contact 215099754@qq.com to obtain it.

Table 2 Surgical Outcomes $(n=8 I)$

\begin{tabular}{|c|c|c|}
\hline & TG & CG \\
\hline & $(n=4 I)$ & $(n=40) P$ \\
\hline Time (min) & & $0.292^{\mathrm{a}}$ \\
\hline Mean \pm SD & $228.97 \pm 7 \mid .27$ & $207.90 \pm 48.84$ \\
\hline Blood loss (mL) & & $0.188^{c}$ \\
\hline Mean $\pm S D$ & $61.71 \pm 55.40$ & $93.25 \pm 156.1$ \\
\hline Surgical approach, n (\%) & & $0.439^{b}$ \\
\hline Laparoscopy & $27(65.9)$ & $23(57.5)$ \\
\hline Robot & $14(34.1)$ & $17(42.5)$ \\
\hline Gastrectomy, n (\%) & & $0.105^{b}$ \\
\hline Distal & $30(73.2)$ & $35(87.5)$ \\
\hline Total & II (26.8) & $5(12.5)$ \\
\hline Type of reconstruction, $\mathrm{n}$ (\%) & & $0.238^{b}$ \\
\hline Roux-en-Y & $36(87.8)$ & $30(75.0)$ \\
\hline Billroth-II & $5(12.2)$ & $10(25.0)$ \\
\hline
\end{tabular}

Notes: ${ }^{\mathrm{a}}$-test; ${ }^{\mathrm{b}} \mathrm{Chi}$-squared test $\left(X^{2}\right) ;{ }^{\mathrm{c}}$ Wilcoxon rank sum test. 
Table 3 Primary Outcomes $(n=81)$

\begin{tabular}{|c|c|c|c|c|}
\hline & \multicolumn{2}{|l|}{ TG } & \multicolumn{2}{|c|}{ CG } \\
\hline & $(n=4 I)$ & & & $=40)$ \\
\hline $\begin{array}{l}\text { PAIN SCORE } \\
\text { Average of POD I-5 } \\
\text { Mean } \pm \text { SD }\end{array}$ & \multicolumn{2}{|c|}{$2.55 \pm 0.21$} & \multicolumn{2}{|c|}{$\begin{array}{l}P<0.001^{b} \\
3.10 \pm 0.42\end{array}$} \\
\hline $\begin{array}{l}\text { POD } 0 \\
\qquad \text { Mean } \pm S D\end{array}$ & \multicolumn{2}{|c|}{$3.58 \pm 0.60$} & \multicolumn{2}{|c|}{$\begin{array}{l}P=0.105^{b} \\
3.83 \pm 0.84\end{array}$} \\
\hline $\begin{array}{l}\text { POD I } \\
\text { Median (Quartile) }\end{array}$ & \multicolumn{2}{|c|}{$3.00(0.42)$} & \multicolumn{2}{|c|}{$3.33(0.67)$} \\
\hline $\begin{array}{l}\text { POD } 2 \\
\quad \text { Median (Quartile) }\end{array}$ & \multicolumn{2}{|c|}{$2.58(0.29)$} & \multicolumn{2}{|c|}{$3.17(0.58)$} \\
\hline $\begin{array}{l}\text { POD } 3 \\
\text { Median (Quartile) }\end{array}$ & \multicolumn{2}{|c|}{$2.25(0.33)$} & \multicolumn{2}{|c|}{$3.08(0.75)$} \\
\hline $\begin{array}{l}\text { POD } 4 \\
\text { Median (Quartile) }\end{array}$ & \multicolumn{2}{|c|}{$2.00(0.17)$} & \multicolumn{2}{|c|}{$2.83(0.58)$} \\
\hline $\begin{array}{l}\text { POD } 5 \\
\text { Median (Quartile) }\end{array}$ & \multicolumn{2}{|c|}{$\mathrm{I} .75(0.2 \mathrm{I})$} & \multicolumn{2}{|c|}{$2.29(0.73)$} \\
\hline $\begin{array}{c}\text { Analysis } \\
\text { Group }\end{array}$ & $\begin{array}{l}\text { Wald Chi- } \\
\text { Square }\end{array}$ & 78.296 & $P$ & $\underset{c}{<0.001}$ \\
\hline Time & $\begin{array}{l}\text { Wald Chi- } \\
\text { Square }\end{array}$ & 419.405 & $P$ & $\underset{c}{<0.001}$ \\
\hline Group*Time & $\begin{array}{l}\text { Wald Chi- } \\
\text { Square }\end{array}$ & 9.155 & $P$ & $0.057^{c}$ \\
\hline ANALGESICS DATA & & & $P$ & \\
\hline $\begin{array}{l}\text { Dose of Flurbiprofen } \\
\text { axetil, } n(\%) \\
\text { Mean } \pm \text { SD }\end{array}$ & $120.0 \pm$ & .82 & & $\begin{array}{l}0.117^{b} \\
.9 \pm 13.22\end{array}$ \\
\hline Use rate of opioids, n (\%) & 18( & & & $\begin{array}{l}0.004^{\mathrm{a}} \\
0(75.0)\end{array}$ \\
\hline $\begin{array}{l}\text { Equivalent dose of } \\
\text { morphine }(\mathrm{mg}) \\
\text { Mean } \pm \text { SD }\end{array}$ & $8.935 \pm$ & & & $\begin{array}{l}0.182^{b} \\
83 \pm 8.097\end{array}$ \\
\hline
\end{tabular}

Notes: ${ }^{a}$ Chi-squared test $\left(X^{2}\right)$; ${ }^{b}$ Wilcoxon rank sum test; ${ }^{\mathrm{c}}$ Generalized estimating equation; $\cdot$ We used an equivalent dose of morphine, pethidine: pentazocine: dezocine: morphine $=100 \mathrm{mg}$ : 45mg: $10 \mathrm{mg}$ : $10 \mathrm{mg}$.

Abbreviation: POD, postoperative day.

\section{Discussion}

Postoperative pain and gastrointestinal dysfunction are two major stumbling blocks that affect the recovery of patients. In addition, as far as pain is concerned, acute and chronic pain that occur during hospitalization and after discharge have caused huge medical and healthcare pressure in European and American countries. ${ }^{18}$ This will also be one of the important issues that China will encounter in the future. In this trial, by comparing the data of POD 1-5, we found that TEAS can relieve the degree of pain by at least $20 \%$ overall, and the decline reached $24.6 \%$ on POD 5. This also caused a reduction in NSAID consumption by $17.8 \%$, opioids consumption by $31.1 \%$, and equivalent dose of morphine by approximately $3.9 \mathrm{mg}$, which meant that the average morphine consumption of patients in TG reduced by nearly half dosage. In terms of gastrointestinal function, it demonstrated a better recovery in TG. In addition, the length of postoperative stay in TG was 0.3 days shorter than $\mathrm{CG}$, though the difference was not statistically significant. Combined with less consumption of analgesics and more optimized perioperative management in the future, it will be effective in reducing the economic burden of patients and hospitals.

Pain management plays a particularly important role in the rehabilitation process. ERAS emphasizes the necessity of effective pain relief targeting the trauma-induced stress. ${ }^{19}$ What's the basis of postoperative pain? Surgery is a nociceptive trigger of tissue or nerve injury. These proinflammatory factors will unbalance various cytokines in microenvironment and then bring about changes in inflammatory immune response which will mediate peripheral and central sensitization, and hence that will affect the body's pain threshold, and cause neuropathic and inflammatory pain, not just mechanical pain. So, there is a close correlation between pain, inflammation and immunomodulation.

According to the theory of TCM, factors such as trauma and anesthesia can lead to Qi and blood obstructed or insufficient, which may cause kinds of pain or visceral dysfunction. Dialectical treatment is the foundation of TCM, and the principles are treating excess with purgation, treating insufficiency with tonification. The acupoints with more similar characteristic are chosen, the better therapeutic effects will achieve. Theoretically, among the selected acupoints in this trial, they are effective in harmonizing meridians and balancing Yin Yang. Hence TEAS with acupoints above took function of enhancing the recovery of patients.

Based on the available evidence, the mechanisms of TEAS analgesia involves several systems such as endorphin system and adrenergic system, in which endorphin system plays a key role. ${ }^{20}$ Previous studies have shown that the release of different endorphins was stimulated by different frequencies and their molecular mechanisms were mediated by different receptors such as $\mu$ and $\kappa$ receptors. $^{21-24} \beta$-End is an endogenous analgesic 

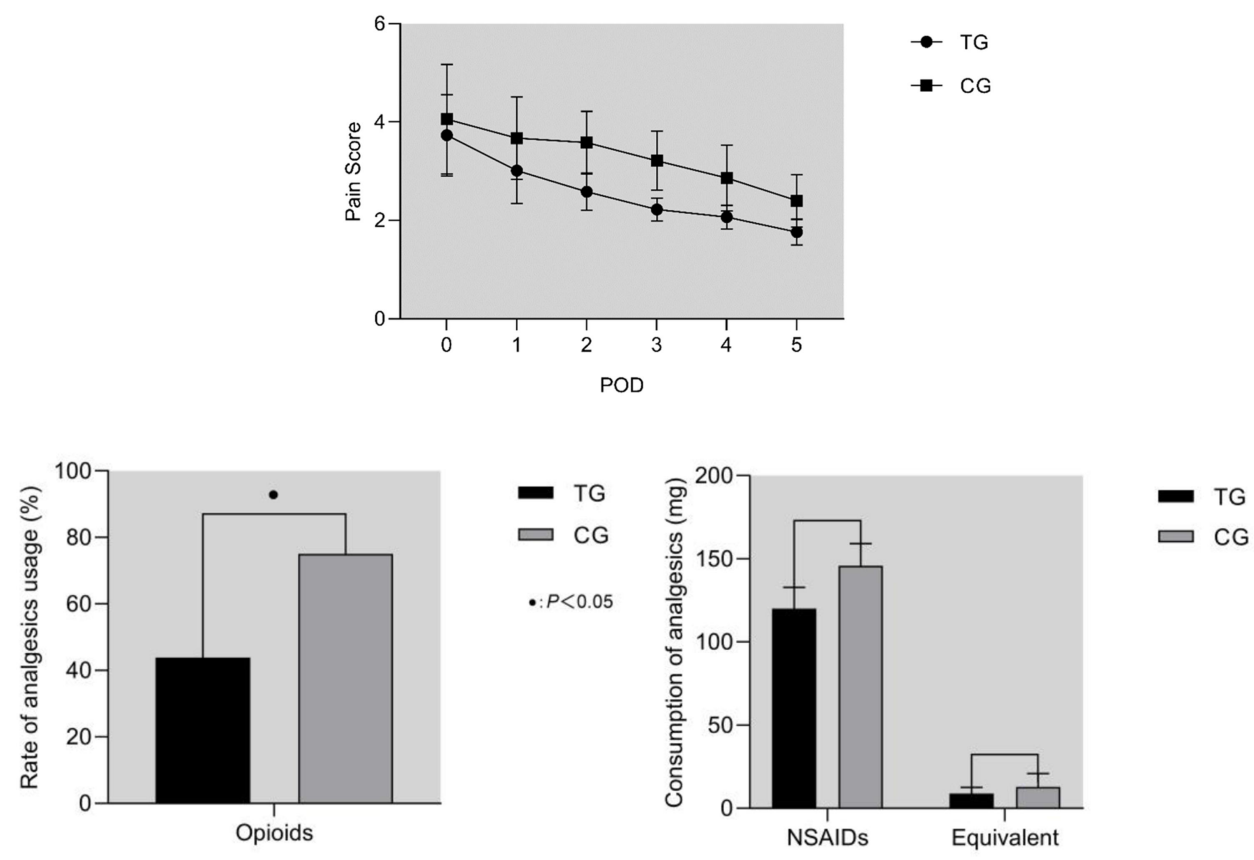

Figure 2 (A) Pain Score. Comparing the pain scores of the two groups, no significant difference was found on POD 0 ( $P=0.105)$. Scores of TG were significantly lower on POD I-5 $(P<0.001)$. (B) Consumption of analgesics. The use rate of opioid was significantly lower in TG $(P=0.004)$. As for the consumption of NSAIDs and the equivalent doses of morphine, no significant difference was found $(P>0.05)$.

Abbreviations: POD, postoperative day; NSAIDs, non-steroidal anti-inflammatory drugs; TG, TEAS group; CG, control group.

substance released by the pituitary chiefly, which alleviate pain through the following aspects: (1) bind to $\mu$ receptor; (2) inhibit the release of transmitters such as substance P to block pathways about pain; ${ }^{22,24}$ (3) block proinflammatory cytokine release and mitigate the extent of neuropathic inflammatory response which will alleviate peripheral and central sensitization, hence it will relieve inflammatory and neuropathic pain. ${ }^{25,26}$ Combined with previous studies and our results, we speculate that the higher endorphin concentration in TG is related to the stimulation of TEAS.

Not only inner analgesics, there were changes in nociception-related factors such as trauma-induced inflammation. TNF- $\alpha$ and IL- 6 both are important indicators to reflect the extent of stress and inflammation. TNF- $\alpha$ is the earliest inflammatory molecule under stress and it participates in the immunomodulation and anti-infection. IL-6 can integrate and cooperate other inflammatory signals to promote inflammation. After the patients suffering from surgical injury, the body will release a variety of nociceptive medias attributing pain. On the one hand, TNF- $\alpha$ and IL- 6 which may overexpress under stress will increase in the injured tissue and central nervous system (CNS), then exacerbate inflammation. On the other hand, these inflammatory molecules also promote the release of phospholipids from tissue, then phospholipids break down into prostaglandins, ${ }^{27}$ which can directly cause pain or intensify the peripheral and central sensitization contributing to pain.

So, superficially it may be another explanation that the anti-nociceptive effects of TEAS may through its regulation of inflammation. Based on what we mentioned above, we think that it may come down to the immunomodulation of TEAS. As we all know, it's extremely common that patients with gastric cancer have immune dysfunction. Stress reactions of varying extent caused by operation, anesthesia and pain will further lead to immunosuppression. ${ }^{28}$ Previous studies have proved that EA has immunomodulatory effect and it can enhance cellular immunity of patients with malignant tumors. ${ }^{29}$ IL-2 plays an important role in promoting lymphocytes maturation and regulating immunity. The production of IL-2 may be inhibited by some negative regulatory molecules which are generated under traumatic stress and hence it results in the decline of immunity. Some scholars have found that EA can promote the production of IL-2, weaken inflammatory factors, and reduce the extent of immunosuppression. ${ }^{29}$ Now it was verified that TEAS has a similar efficacy on protecting the immunity. In mechanisms, there may be a cholinergic anti-inflammatory pathway and hypothalamic-pituitary-adrenal axis involved 
Table 4 Secondary Outcomes $(n=8 I)$

\begin{tabular}{|c|c|c|}
\hline & TG & CG \\
\hline & $(n=41)$ & $(n=40) P$ \\
\hline Time of first flatus (h) & & $<0.00 \mathrm{I}^{\mathrm{b}}$ \\
\hline Mean $\pm S D$ & $55.63 \pm 16.74$ & $72.60 \pm 20.92$ \\
\hline Time of first defecation (h) & & $<0.001^{b}$ \\
\hline Mean \pm SD & $72.20 \pm 16.24$ & $95.78 \pm 17.75$ \\
\hline Nausea, n (\%) & & $0.44 I^{a}$ \\
\hline & $16(39.0)$ & $19(47.5)$ \\
\hline Vomiting, n (\%) & & $1.00^{\mathrm{a}}$ \\
\hline & $0(0.00)$ & $0(0.00)$ \\
\hline Distension, n (\%) & & $0.05 \mathrm{I}^{\mathrm{a}}$ \\
\hline & $10(24.4)$ & $18(45.0)$ \\
\hline Diarrhea, n (\%) & & $0.054^{\mathrm{a}}$ \\
\hline & $3(7.30)$ & $9(22.5)$ \\
\hline Fever, n (\%) & & $0.753^{a}$ \\
\hline & $36(87.8)$ & $36(90.0)$ \\
\hline Frequency of nausea & & $0.029^{b}$ \\
\hline Mean \pm SD & $1.88 \pm 1.09$ & $2.58 \pm 0.77$ \\
\hline Frequency of vomiting & & $1.00^{b}$ \\
\hline Mean \pm SD & 0 & 0 \\
\hline Frequency of distension & & $0.213^{b}$ \\
\hline Mean \pm SD & $2.60 \pm 1.27$ & $3.50 \pm 2.01$ \\
\hline Frequency of diarrhea & & $0.727^{b}$ \\
\hline Mean \pm SD & $2.00 \pm 1.00$ & $2.33 \pm 1.12$ \\
\hline Frequency of fever & & $0.419^{b}$ \\
\hline Mean \pm SD & $6.35 \pm 4.03$ & $7.61 \pm 6.13$ \\
\hline Comfort of semi-fluid & & $<0.001^{b}$ \\
\hline Mean \pm SD & $7.63 \pm 0.63$ & $6.93 \pm 0.69$ \\
\hline C-D grade, $n$ (\%)• & & $0.292^{\mathrm{a}}$ \\
\hline Grade 0 & I (2.40) & $2(5.00)$ \\
\hline Grade I & $39(95.1)$ & $34(85.0)$ \\
\hline Grade 2 & I (2.40) & $4(10.0)$ \\
\hline Length of postoperative stay (d) & & $0.478^{b}$ \\
\hline Mean \pm SD & $6.12 \pm 1.29$ & $6.45 \pm 1.68$ \\
\hline
\end{tabular}

Notes: a Chi-squared test $\left(X^{2}\right)$; ${ }^{b}$ Wilcoxon rank sum test; $\bullet$ The $C$-D grade was used for postoperative complications grade; Patients with a grade $>2$ met the withdrawal criteria and were not included in the analysis.

Abbreviation: C-D grade, Clavien-Dindo grade.

in the inflammatory reflex to achieve immune homeostasis. ${ }^{25}$ And some studies have found that EA may regulate neuroimmune reflex and inhibit inflammation by modulating dopamine in mouse models of sepsis. ${ }^{5}$ Moreover, immunity and endorphin system can interact on each other. ${ }^{29,30}$ Overall, pain, inflammation, and immunity are interrelated and mutually reinforcing.

Postoperative gastrointestinal dysfunction may occur because of surgical operation (damage of vagal nerve, changes in physiological structure), $\mathrm{CO}_{2}$ pneumoperitoneum (ischemia caused by abdominal hypertension), etc. MTL is an important hormone which can promote gastrointestinal transmission and strengthen esophageal sphincter contraction to prevent gastric reflux. Emetic molecule such as 5-HT which will increase under trauma or anesthesia, can directly stimulate the vomiting center of medulla oblongata or activate the peripheral receptors in the gastrointestinal mucosa. ${ }^{31}$ From our results, we considered that TEAS promote gastrointestinal motility and reduce the incidence of nausea by regulating MTL and 5-HT. TEAS may affect 5-HT transmission by activating 5-HT and norepinephrine fibers, and TEAS may also regulate the vagal reflex to decrease the release of 5-HT in the center. Thus, the binding rate of 5-HT in gastrointestinal tract and peripheral nerve reduced. ${ }^{32}$ However, there was no difference in vomiting between 2 groups. We considered that it was related to the implementation of ERAS for its early enteral feeding and mobilization. In addition, postoperative pain and the use of opioids will further prolong the recovery of gastrointestinal function. ${ }^{33}$ So, a better analgesia will bring about a faster recovery. And these are the reasons why the patients in TG had a higher comfort of semi-fluid.

There were some limitations in this trial: (1) We did not collect and stratify the social class, personality characteristics which may cause unknown bias. (2) About secretion of transmitters such as endorphins, there is a circadian pattern in the body which may lead to a biological rhythm of pain. So, time deviation may exist in the process of collecting clinical data. (3) In the analysis of consumption of opioids, for pentazocine, the equivalent dose of morphine is 30 to $60 \mathrm{mg}$. Due to individual differences in pharmacodynamics, we have not found a clear equivalent dose for the Chinese constitution. (4) $2 / 100 \mathrm{~Hz}$ mode is more common in other trials, and the reasons why we choose $20 / 100 \mathrm{~Hz}$ are as follows. First, the frequency of $15-30 \mathrm{~Hz}$ is more effective in promoting the release of oxytocin than $2-3 \mathrm{~Hz}$, and oxytocin can also relieve pain and enhance the analgesic effect of electroacupuncture. Second, $15 \mathrm{~Hz}$ can both promote the release of $\beta$-End and Enk. Although it is not as effective as $2 \mathrm{~Hz}$ in releasing $\beta$-End, ${ }^{21}$ this mode still can be used. 
Table 5 Hematological Results (TG=4I; CG=40)

\begin{tabular}{|c|c|c|c|c|c|}
\hline & POD I & POD 3 & POD5 & Analysis Wald Chi-Square (Group) \\
\hline & & Median (Quartile) & Median (Quartile) & Median (Quartile) & $P$ \\
\hline \multirow[t]{2}{*}{ WBC $\left(10^{9} / \mathrm{L}\right)$} & TG & $10.64(3.735)$ & $7.320(2.625)$ & $6.480(1.590)$ & 0.571 \\
\hline & CG & $12.15(4.668)$ & $7.190(3.678)$ & $6.425(2.850)$ & $0.450^{d}$ \\
\hline \multirow[t]{2}{*}{ NEC\% } & TG & $83.10(9.650)$ & $73.90(10.45)$ & $69.80(5.400)$ & 0.070 \\
\hline & CG & $83.95(12.83)$ & $75.25(9.275)$ & $67.60(9.675)$ & $0.791^{d}$ \\
\hline \multirow[t]{2}{*}{ CRP (mg/L) } & TG & $14.74(12.86)$ & 61.71 (34.26) & 27.33 (23.7I) & 2.909 \\
\hline & CG & $21.00(13.57)$ & $67.57(37.69)$ & $31.54(24.40)$ & $0.088^{d}$ \\
\hline \multirow[t]{2}{*}{ PCT (ng/mL) } & TG & $0.103(0.111)$ & $0.255(0.180)$ & $0.092(0.052)$ & 2.853 \\
\hline & CG & $0.112(0.104)$ & $0.275(0.258)$ & $0.136(0.117)$ & $0.091^{d}$ \\
\hline \multirow[t]{2}{*}{$\beta$-End ( $\mathrm{pg} / \mathrm{mL})$} & TG & $49.16(8.08)$ & $73.92(6.38)$ & $68.20(2.450)$ & 467.661 \\
\hline & CG & $50.53(6.11)$ & $59.48(4.46)$ & $53.31(4.070)$ & $<0.001^{d}$ \\
\hline \multirow[t]{2}{*}{ 5-HT (pg/mL) } & TG & $2137.82(353.19)$ & $1607.72(272.86) \mid 852.97(295.24)$ & $1330.9 \mid(459.38)$ & 28.013 \\
\hline & CG & 2049.37 (176.22) & & $1556.23(359.15)$ & $<0.001^{d}$ \\
\hline \multirow[t]{2}{*}{ IL-2 (ng/mL) } & TG & $4.740(1.210)$ & $7.810(2.640)$ & $9.590(3.190)$ & 52.534 \\
\hline & CG & $4.535(1.420)$ & $6.295(0.800)$ & $7.665(1.050)$ & $<0.001^{d}$ \\
\hline \multirow[t]{2}{*}{ IL-6 (pg/mL) } & TG & $45.4 I(7.690)$ & $39.17(12.57)$ & $31.83(4.270)$ & 33.888 \\
\hline & CG & $46.08(7.800)$ & $42.46(3.520)$ & $35.60(2.260)$ & $<0.001^{d}$ \\
\hline \multirow[t]{2}{*}{ TNF- $\alpha$ (pg/mL) } & TG & $48.53(5.700)$ & 36.89 (10.99) & $27.53(5.700)$ & 20.734 \\
\hline & CG & $48.55(6.770)$ & $41.38(6.690)$ & $31.12(4.620)$ & $<0.001^{d}$ \\
\hline \multirow[t]{2}{*}{ MTL (pg/mL) } & TG & 392.44 (127.48) & 522.78 (127.7I) & $647.68(157.06)$ & 36.611 \\
\hline & CG & 397.45 (133.54) & $486.90(68.840)$ & 556.70 (93.69) & $<0.001^{d}$ \\
\hline
\end{tabular}

Note: ${ }^{\mathrm{d}}$ Generalized estimating equation.

Abbreviations: WBC, white blood cell count; NEC\%, percentage of neutrophils; CRP, C-reactive protein; PCT, procalcitonin; $\beta$-End, $\beta$-endorphin; 5-HT, 5 -hydroxytryptamine; IL-2, interleukin-2; IL-6, interleukin-6; TNF- $\alpha$, tumor necrosis factor- $\alpha$; MTL, motilin.

\section{Conclusion}

In Summary, it was confirmed that TEAS played a role in relieving postoperative pain and promoting the recovery of gastrointestinal function, which may be related to the modulation of inflammation. And there was no adverse reaction during the entire trial. Therefore, TEAS can be used as an adjuvant therapy to enhance the postoperative recovery for patients with gastric cancer.

\section{Disclosure}

We declare that we do not have any commercial or associative interest that represents a conflict of interest in connection with the work submitted. And informed consents have been obtained from all patients included in this trial.

\section{References}

1. Bray F, Ferlay J, Soerjomataram I, Siegel RL, Torre LA, Jemal A. Global cancer statistics 2018: GLOBOCAN estimates of incidence and mortality worldwide for 36 cancers in 185 countries. CA Cancer J Clin. 2018;68:394-424. doi:10.3322/caac.21492
2. Kim HH, Han SU, Kim MC, et al. Long-term results of laparoscopic gastrectomy for gastric cancer: a large-scale case-control and case-matched Korean multicenter study. J Clin Oncol. 2014;32:627-633. doi:10.1200/JCO.2013.48.8551

3. Gerbershagen HJ, Aduckathil S, van Wijck AJ, Peelen LM, Kalkman CJ, Meissner W. Pain intensity on the first day after surgery: a prospective cohort study comparing 179 surgical procedures. Anesthesiology. 2013;118:934-944. doi:10.1097/ALN.0b013e31828866b3

4. Wu CL, Raja SN. Treatment of acute postoperative pain. Lancet. 2011;377:2215-2225. doi:10.1016/S0140-6736(11)60245-6

5. Stein C, Schäfer M, Machelska H. Attacking pain at its source: new perspectives on opioids. Nat Med. 2003;9:1003-1008. doi:10.1038/nm908

6. Kehlet H. Multimodal approach to control postoperative pathophysiology and rehabilitation. $B r \quad J$ Anaesth. 1997;78:606-617. doi:10.1093/bja/78.5.606

7. Aarts MA, Rotstein OD, Pearsall EA, et al. Postoperative eras interventions have the greatest impact on optimal recovery: experience with implementation of eras across multiple hospitals. Ann Surg. 2018;267:992-997. doi:10.1097/SLA.0000000000002632

8. Ljungqvist O, Scott M, Fearon KC. Enhanced recovery after surgery: a review. JAMA Surg. 2017;152:292-298. doi:10.1001/jamasurg.2016.4952

9. Kehlet H. Postoperative pain, analgesia, and recovery-bedfellows that cannot be ignored. Pain. 2018;159(Suppl 1):S11-S16. doi:10.1097/j. pain.0000000000001243

10. Wick EC, Grant MC, Wu CL. Postoperative multimodal analgesia pain management with nonopioid analgesics and techniques: a review. JAMA Surg. 2017;152:691-697. doi:10.1001/jamasurg.20 17.0898 
11. Patil S, Sen S, Bral M, et al. The role of acupuncture in pain management. Curr Pain Headache Rep. 2016;20:22. doi:10.1007/ s11916-016-0552-1

12. Tang J-L, Liu B-Y, Ma K-W. Traditional Chinese medicine. Lancet. 2008;372:1938-1940. doi:10.1016/S0140-6736(08)61354-9

13. Ulett GA, Han S, Han JS. Electroacupuncture: mechanisms and clinical application. Biol Psychiatry. 1998;44:129-138. doi:10.1016/ S0006-3223(97)00394-6

14. Wang H, Xie Y, Zhang Q, et al. Transcutaneous electric acupoint stimulation reduces intra-operative remifentanil consumption and alleviates postoperative side-effects in patients undergoing sinusotomy: a prospective, randomized, placebo-controlled trial. $\mathrm{Br}$ $J$ Anaesth. 2014;112:1075-1082. doi:10.1093/bja/aeu001

15. Zhang Q, Gao Z, Wang $H$, et al. The effect of pre-treatment with transcutaneous electrical acupoint stimulation on the quality of recovery after ambulatory breast surgery: a prospective, randomised controlled trial. Anaesthesia. 2014;69:832-839. doi:10.1111/anae.12639

16. Zhang B, Xu F, Hu P, et al. Needleless transcutaneous electrical acustimulation: a pilot study evaluating improvement in post-operative recovery. Am J Gastroenterol. 2018;113 (7):1026-1035. doi:10.1038/s41395-018-0156-y

17. Yu X, Zhang F, Chen B. The effect of TEAS on the quality of early recovery in patients undergoing gynecological laparoscopic surgery: a prospective, randomized, placebo-controlled trial. Trials. 2020;21 (1):43. doi:10.1186/s13063-019-3892-4

18. Johnson MI, Jones G, Paley CA, Wittkopf PG. The clinical efficacy of transcutaneous electrical nerve stimulation (TENS) for acute and chronic pain: a protocol for a meta-analysis of randomised controlled trials (RCTs). BMJ Open. 2019;9(10):e029999. doi:10.1136/bmjopen-2019-029999

19. Hughes MJ, Ventham NT, McNally S, Harrison E, Wigmore S. Analgesia after open abdominal surgery in the setting of enhanced recovery surgery: a systematic review and meta-analysis. JAMA Surg. 2014;149:1224-1230. doi:10.1001/jamasurg.2014.210

20. Han JS. Acupuncture and endorphins. Neurosci Lett. 2004;361:258-261. doi:10.1016/j.neulet.2003.12.019

21. Han JS. Acupuncture: neuropeptide release produced by electrical stimulation of different frequencies. Trends Neurosci. 2003;26:17-22. doi:10.1016/S0166-2236(02)00006-1
22. Zhao ZQ. Neural mechanism underlying acupuncture analgesia. Prog Neurobiol. 2008;85:355-375.

23. Wang Y, Zhang Y, Wang W, Cao Y, Han JS. Effects of synchronous or asynchronous electroacupuncture stimulation with low versus high frequency on spinal opioid release and tail flick nociception. Exp Neurol. 2005;192:156-162. doi:10.1016/j.expneurol.2004.11.003

24. Groppetti D, Pecile AM, Sacerdote P, Bronzo V, Ravasio G. Effectiveness of electroacupuncture analgesia compared with opioid administration in a dog model: a pilot study. $\mathrm{Br} J$ Anaesth. 2011;107:612-618. doi:10.1093/bja/aer199

25. Lin JG, Chen WL. Acupuncture analgesia: a review of its mechanisms of actions. Am J Chin Med. 2008;36:635-645. doi:10.1142/ S0192415X08006107

26. Zhang R, Lao L, Ren K, et al. Mechanisms of acupuncture-electroacupuncture on persistent pain. Anesthesiology. 2014;120:482-503. doi:10.1097/ALN.0000000000000101

27. Alazawi W, Pirmadjid N, Lahiri R, Bhattacharya S. Inflammatory and immune responses to surgery and their clinical impact. Ann Surg. 2016;264:73-80. doi:10.1097/SLA.0000000000001691

28. Carr DB, Goudas LC. Acute pain. Lancet. 1999;353:2051-2058. doi:10.1016/S0140-6736(99)03313-9

29. Cabioğlu MT, Cetin BE. Acupuncture and immunomodulation. Am $J$ Chin Med. 2008;36:25-36. doi:10.1142/S0192415X08005552

30. Stein C. The control of pain in peripheral tissue by opioids. $N$ Engl J Med. 1995;332:1685-1690. doi:10.1056/NEJM199506223322506

31. Janicki PK, Sugino S. Genetic factors associated with pharmacotherapy and background sensitivity to postoperative and chemotherapy-induced nausea and vomiting. Exp Brain Res. 2014;232:2613-2625. doi:10.1007/s00221-014-3968-Z

32. Lee S, Lee MS, Choi DH, Lee SK. Electroacupuncture on pc6 prevents opioid-induced nausea and vomiting after laparoscopic surgery. Chin J Integr Med. 2013;19:277-281. doi:10.1007/s11655013-1425-7

33. Ng SS, Leung WW, Mak TW, et al. Electroacupuncture reduces duration of postoperative ileus after laparoscopic surgery for colorectal cancer. Gastroenterology. 2013;144:307-313 e1. doi:10.1053/j. gastro.2012.10.050

\section{Publish your work in this journal}

Cancer Management and Research is an international, peer-reviewed open access journal focusing on cancer research and the optimal use of preventative and integrated treatment interventions to achieve improved outcomes, enhanced survival and quality of life for the cancer patient.
The manuscript management system is completely online and includes a very quick and fair peer-review system, which is all easy to use. Visit http://www.dovepress.com/testimonials.php to read real quotes from published authors. 